\title{
REFLECTANCE METHOD FOR A WEARABLE PHOTOPLETHYSMOGRAPHIC SENSOR - EXPERIMENTAL EVALUATION
}

\author{
Tadeusz SONDEJ', Krzysztof RÓŻANOWSKI', Dominik SONDEJ' \\ ${ }^{1}$ Military University of Technology, Electronic Department, Warsaw, Poland \\ ${ }^{2}$ Military Institute of Aviation Medicine, Aviation Bioengineering Department, Warsaw, Poland
}

Source of support: This work was supported in part by the Polish National Centre for Research and Development under project No. DOBR/0038/R/ID2/2013/03.

Author's address: T. Sondej, Military University of Technology, Electronic Department, Gen. S. Kaliskiego 2 Street, 00-908 Warsaw 49, Poland; e-mail: tadeusz.sondej@wat.edu.pl

Introduction: The article presents the design and testing results of a photoplethysmographic signal sensor intended for use in everyday activities.

Methods: The design of the sensor involved a commercial DCM03 sensor based on the reflectance method and a fully integrated analog, front-end AFE4490. Determination of the photoplethysmographic signal and calculation of heart rate was based on appropriate data processing algorithms including digital filters, signal segmentation, moving average and variable baseline.

Results: Measurements were carried out in different sensor placement locations as well as during specific body motions. Detailed analysis of the recorded signal was performed using the proposed processing methods and the fast Fourier transform technique.

Discussion: Based on our results, it is possible to measure HR values and determine the photoplethysmographic curve under dynamic conditions. On the other hand measurement of blood oxygen saturation is subject to significant limitations.

Keywords: pulse oximetry, reflectance sensor, photoplethysmography, wearable sensor, PPG

Figures: 29 - Tables: 2 - References: 33 - Full-text PDF: http://www.pjambp.com • Copyright @ 2016 Polish Aviation Medicine Society, ul. Krasińskiego 54/56, 01-755 Warsaw, license WIML • Indexation: Index Copernicus, Polish Ministry of Science and Higher Education 


\section{INTRODUCTION}

Recent years have witnessed a considerable progress in the development of mobile devices for the measurement of heart rate (HR) and blood oxygen saturation $\left(\mathrm{SpO}_{2}\right)$, based on photoplethysmographic (PPG) reflectance sensors. This has been associated with advancements in telemedicine and the need to monitor psychophysiological state of subjects under dynamic conditions. The main problem in such measurements involves providing an appropriate quality of the PPG signal which is prone to interference caused by movement. Different sensor designs, placement locations and data processing methods have been attempted, with designers intending to facilitate acquisition of correct measurements under dynamic conditions without disturbing everyday functioning.

Photoplethysmography is currently the most popular technique used for the measurement of blood oxygen $\left(\mathrm{O}_{2}\right)$ content. It is commonly referred to as pulse oximetry. The method involves the use of two wavelengths to determine arterial blood absorbance that correlates with blood oxygen levels. Over the years, measurements of blood oxygen saturation $\left(\mathrm{SpO}_{2}\right)$ or just of heart rate $(\mathrm{HR})$, with the use of pulse oximeters, became vital parameters used not only for purely medical applications but in sports and telemedicine as well. Numerous embodiments of commercial devices for the measurement of $\mathrm{SpO}_{2}$ are available. However, most of these devices are based on the transmission method, with the light emitter and receiver being placed on opposite sides of chosen body parts, most commonly on the index finger. This measurement method restricts the range of motion and is not suitable for all-day use. Therefore, the reflectance method involving side-by-side placement of a light emitter and receiver provides a better solution for continuous processing. Implementation of this method was described in [1] as well as in many other reports. Nonetheless, improvements in the reflectance method, particularly with regard to operation under dynamic conditions and minimization of energy consumption, continue to be a topical matter. Development of efficient methods of correcting motion-related artifacts is currently the biggest problem in reflectance pulse oximetry. The aim of the article is to present the state of knowledge on photoplethysmographic signal measurements used in the reflectance method and an experimental evaluation of an application of this method to build a wearable sensor. The article also presents the operation of the developed sensor that was placed in different body parts and during performance of specific body movements.

Sensor placement location is a very important factor in reflectance measurements. Many measurement sites are found in the literature, including the forehead [2], the wrist [3], the finger [4], the back [5], the earlobe and even the ear canal [6]. Unfortunately, not all of these locations are suitable for use during everyday activities. Many attempts have been made to improve the design of measurement devices. Typical custom solutions include an amplifier, filters and an A/C transducer, [e.g. $3,6,7,8,9]$, but attempts to use smartphones [10] or FPGA systems [11] have also been made. In this study, a dedicated, integrated circuit was used. A more detailed review of solutions applied in wearable photoplethysmographic sensors is provided in [12]. The article focuses on the methods of data processing compensating for motionassociated disturbances.

Clearly, monitoring of people under dynamic conditions is significantly different from that under stationary conditions. Dynamic conditions are associated with the presence of additional problems due to the movements of the monitored subject. The main problems include motion-related artifacts and reduced supply of energy required to power the measurement system. Subject's motion negatively affects operation of all types of pulse oximeters. This is particularly evident in measurements based on the reflectance methods. Pulse oximeter sensors are placed in contact with human skin. Usually, they are lightly pressed against the skin; the pressure should not be excessive so as not to cause increased temperature and perspiration under the sensor. If the monitored patient moves, the contact between the skin and the sensor is disturbed and the pressure against the blood vessels is changed. This leads to a disturbance or disappearance of the PPG signal, significantly hampering determination of correct $\mathrm{SpO}_{2}$ values [13].

Much of the available literature focuses on elimination of motion-related artifacts and recovery of the clear PPG waveform by means of appropriate processing of the disturbed signal. Disturbances due to the patient's motion are usually random in character, with a mean frequency similar to that of the PPG signal, and therefore the elimination of these disturbances is very difficult. Numerous complex data processing methods are used in order to reduce the effect of motion-related disturbances on the PPG signal. Relevant digital filters or Fourier series analysis may be applied on a cycle-by-cycle 
basis $[14,15]$. The method allows for the recovery of a disturbed PPG signal with the accuracy of $5 \%$; however, it is insensitive to the changes in heart rate. A moving average method is also used [16]. The method is based on a quasi-periodicity of the PPG signal, with motion-related artifacts being eliminated by appropriate averaging of PPG signal samples. This method is also characterized by a poor sensitivity to the changes in heart rate. The disturbed PPG signal may also be processed by means of wavelet analysis [17] or independent component analysis (ICA) [18]. Motion-related disturbances may also be reduced by using an accelerometer signal to control the adaptive filter [19]. An article [20] proposed an adaptive normalized least mean square (NLMS) algorithm to obtain a high correlation (0.98 for HR and 0.7 for SpO2) between the reference signal and the signal processed according to the algorithm; however, the studies used a transmission sensor. A high efficacy of elimination of disturbances in PPG measurements was achieved with the method proposed by Masimo Corporation [21] and with its modification [22]. The Masimo method involved a discrete saturation transform (DST) algorithm. Another article [23] describes the design of a reflectance sensor placed on the earlobe together with data processing including Bland-Altman and correlation analysis. A high correlation was achieved between the GR values calculated by the proposed method and those measured from a reference ECG signal. Correlation coefficients were 0.97 in the standing position, 0.82 while walking and 0.76 while running, respectively. Another method to reduce the impact of motion-related disturbances is the method of the ratios between the absorption of red and infrared light described in [24].

\section{PRINCIPLE OF PHOTOPLETHYSMOGRAPHY}

\section{Measurement principle}

Oxygen saturation of arterial blood $\mathrm{SaO}_{2}$ is defined as a percentage parameter calculated by the following formula:

$$
\frac{\mathrm{HbO}_{2}}{\mathrm{Hb}+\mathrm{HbO}_{2}+\mathrm{HbCO}+\mathrm{MetHb}+\text { other hemoglobins }} \times 100
$$

It is often considered equivalent to the result of the measurement carried out using a pulse oximeter, which is referred to as $\mathrm{SpO}_{2}$. However, the result may be inaccurate when the patient has high quantities of hemoglobin incapable of transporting oxygen (e.g. carboxyhemoglobin $\mathrm{HbCO}$ or methemoglobin MetHb) [15]. This is because pulse oximetry measurements are based on the assumption that the arterial blood is free of hemoglobin incapable of transporting oxygen with blood oxygenation status defined as:

$$
\frac{\mathrm{HbO}_{2}}{\mathrm{Hb}+\mathrm{HbO}_{2}} \times 100
$$

In most cases, the amounts of $\mathrm{HbCO}$ and MetHb are small enough for $\mathrm{SaO}_{2}$ to be approximated by $\mathrm{SpO}_{2}$. However, upon intoxication with carbon monoxide or heavy habitual smoking, the quantity of $\mathrm{HbCO}$ is significantly increased, leading to a marked difference between both parameters. In a healthy human, $\mathrm{SpO}_{2}$ is assumed to be at the level of $97-100 \%$.

The measurements of $\mathrm{SpO}_{2}$ are based on light absorption differences between $\mathrm{HbO}_{2}$ and $\mathrm{Hb}$ for different wavelengths. Fig. 1 presents the relationship between the molar absorption coefficients for $\mathrm{HbO}_{2}$ and $\mathrm{Hb}$ and the wavelength within the visible red and infrared light region. As seen in the presented spectrum, $\mathrm{Hb}$ and $\mathrm{Hb} 2 \mathrm{O}$ are characterized by different absorbance throughout the spectral range with the exception of the crossing point $(805 \mathrm{~nm})$ where the absorption coefficients are equal.

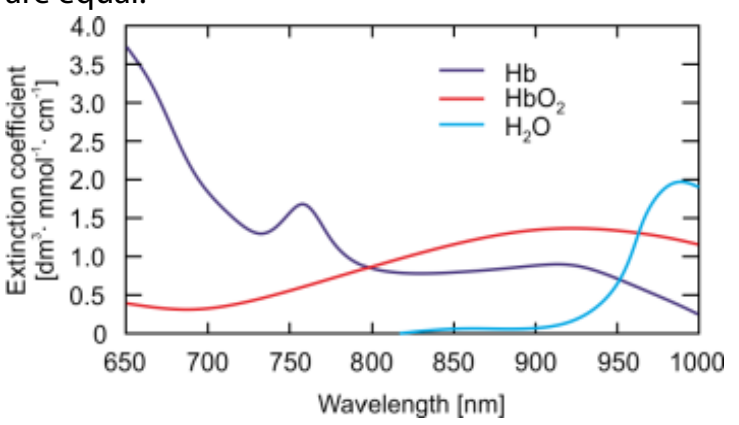

Fig. 1. Absorption spectrum for $\mathrm{Hb}$ and $\mathrm{HbO}_{2}$.

For the wavelengths of less than $805 \mathrm{~nm}, \mathrm{Hb}$ has a higher absorption coefficient as compared to $\mathrm{HbO}_{2}$, while the opposite is true for the wavelengths above $805 \mathrm{~nm}$. In most cases, frequencies used in pulse oximeters include $660 \mathrm{~nm}$ (red light) and $940 \mathrm{~nm}$ (infrared light). The principal law applicable to pulse oximetry measurements is the Beer-Lambert law that describes the absorption of light transmitted through the tissue.

Periodic changes in blood vessel frequency due to the activity of the heart affect the intensity of light being received. The changes of light intensity over time are referred to as photoplethysmographic (PPG) curve. Light is scattered in many directions, with the forward direction being predominant. Therefore, the light passing through 
the tissue is characterized by the highest changes in intensity. In the case of reflectance pulse oximeters, however, changes in the intensity of light being scattered backwards are of the highest interest. A great advantage of this type of pulse oximeters is that they require only one surface.

In practice, $\mathrm{SpO}_{2}$ is determined from the optical density ratio $\mathrm{R}$ [25] calculated from formula (3):

$$
R=\frac{\ln \left(\frac{I_{M A X}\left(\lambda_{r}\right)}{I_{M I N}\left(\lambda_{r}\right)}\right)}{\ln \left(\frac{I_{M A X}\left(\lambda_{i r}\right)}{I_{M I N}\left(\lambda_{i r}\right)}\right)}
$$

where:

$\left(\frac{I_{M A X}\left(\lambda_{r}\right)}{I_{M A X}\left(\lambda_{i r}\right)}\right) \begin{aligned} & \text { - is the value of the photoplethy- } \\ & \text { smographic signal for the RED and }\end{aligned}$ IR diodes at a local maximum within a particular segment,

$\left(\frac{I_{M I N}\left(\lambda_{r}\right)}{I_{M I N}\left(\lambda_{i r}\right)}\right) \begin{aligned} & - \text { is the value of the signal at a local } \\ & \text { minimum. }\end{aligned}$

When the normalized $\mathrm{R}$ ratio is known, $\mathrm{SpO}_{2}$ may be determined from an empirical relationship between $\mathrm{SpO}_{2}$ and $\mathrm{R}$ described by formula (4) [25].

$$
\mathrm{SpO}_{2}=\frac{0,81-0,18 \times R}{0,63+0,11 \times R}
$$

In most cases, however, the value of $\mathrm{SpO}_{2}$ calculated by empirical means is different from the actual value and thus a relationship described by formula (5) may be encountered more often, with $a$ and $b$ being constants determined during calibration.

$$
\mathrm{SpO}_{2}=a-b \times R
$$

\section{Transmission and reflectance sensors}

The PPG signal may be obtained by two distinct methods: transmission and reflectance, both illustrated in Fig. 2. In the transmission methods, the light source and the photodetector are placed on opposite sides of the medium (Fig. 2a).

A clip may be used to compress both devices to the medium, preventing dislocation of the light a)

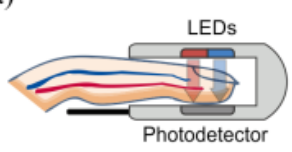

b)

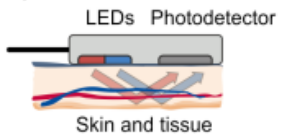

Fig. 2. Measurement of PPG signals using the transmission (a) and reflectance method (b). sources or the photodetector during measurements. However, when the pressure is too strong, it may cause perspiration and elevated temperature of the skin under the sensor. An advantage of the transmission sensor is the ability to obtain a strong and good quality PPG signal and a fast placement method. However, it may be applied only to selected body parts such as fingers, ears or feet. Moreover, the signal is disturbed by daily activities.

The use of reflectance sensors, with light sources being placed on the same side as the photodetector, may provide a solution to problems associated with the placement of the transmission sen-

sors (Fig. 2b). Motion-related artifacts and blood pressure disturbances have a significant and negative effect on the measurements in these type of sensors. Numerous reflectance sensors in various configurations are encountered in the literature $[2,8,16,26]$. The most popular (Fig. 3) include sensors consisting of individual photo-emitters (of red and infrared light) and one [9,27,28] (Fig. 3 b, c, e) or more detectors [3] (Fig. $3 \mathrm{a}, \mathrm{d}$ ).

A method of placing numerous detectors as

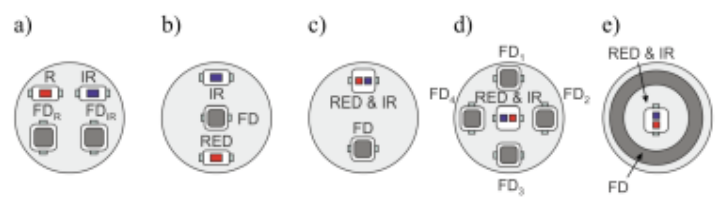

Fig. 3. Example placements of red (R, RED) and infrared (IR) light sources and photodetectors (FD)on the surface of tissue.

a ring around the arm is also known [7]. However, these are only model solutions that are not available on the market. Moreover, they are characterized by large external dimensions. Therefore, the manufacturers of electronic devices have also developed some adapted solutions. The most popular commercial solutions include integrated sensors consisting of emitter diodes and a receiver photodiode (APM Korea, OSRAM Opto Semiconductors) or phototransistor (New Japan Radio). These solutions provide an alternative to custom sensors. They are small, widely available and inexpensive. Another important feature is the repeatability of the obtained optical parameters. There-

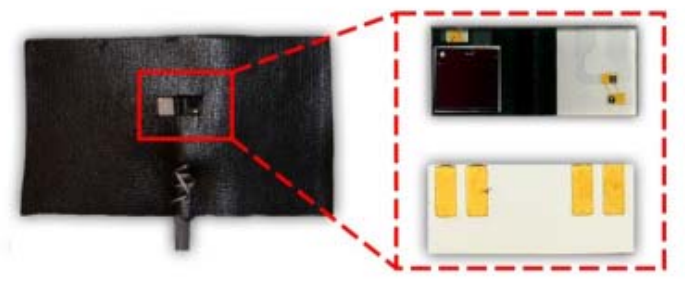

Fig. 4. A board with a DMC03 sensor. 


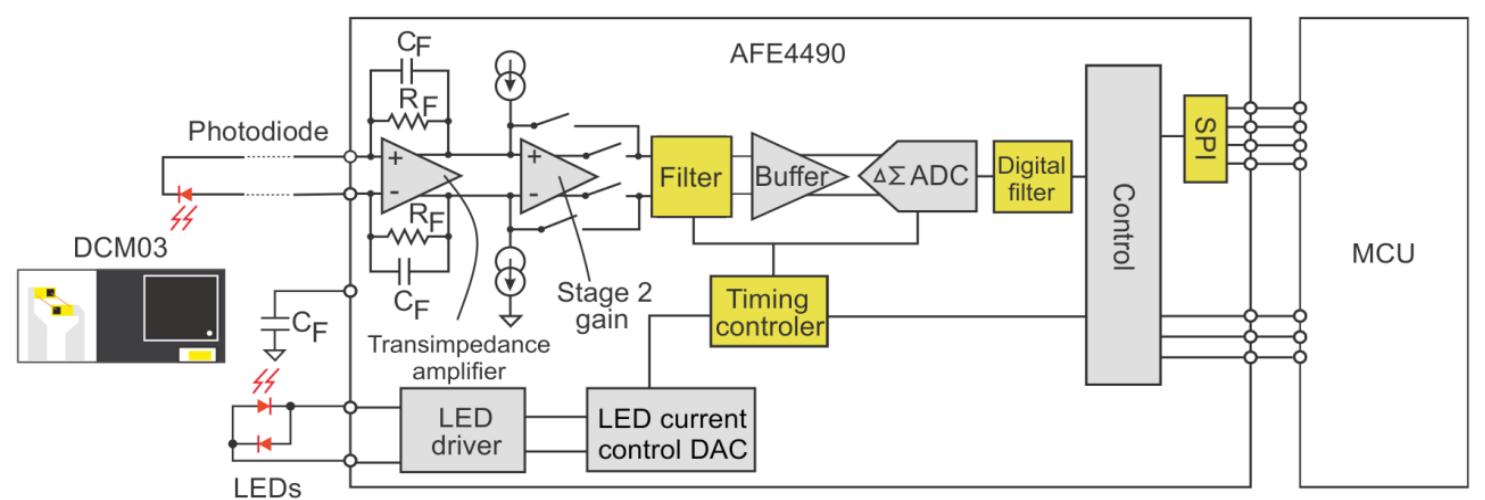

Fig. 5. Block diagram of a measurement system with DCM03 sensor.

fore, the development of a custom wearable pulse oximeter was based on a commercial DCM03 sensor from APM Korea [29].

\section{Sensor design}

The reflectance sensor DCM03 was placed on a small printed circuit board (Fig. 4) and connected to the measurement system by means of a cable (ca. $1 \mathrm{~m}$ long).

The dimensions of the DCM03 are $9.8 \mathrm{~mm} \times 4.3$ $\mathrm{mm}$; it features two integrated LEDs with emission wavelengths of $660 \mathrm{~nm}$ (red) and $905 \mathrm{~nm}$ (infrared) as well as a photodiode with a spectral range of $400-1050 \mathrm{~nm}$. Fig. 5 presents a block diagram of the custom measurement system.

The designed measurement system features an integrated oxygen saturation controller AFE4490 [30] from Texas Instruments. AFE4490 is a fully integrated analog front-end for pulse oximeter applications. It consists of LED driver systems, a photodetector amplifier, filters, A/C converter and a serial peripheral interface (SPI). The emitter diodes (RED and IR) of the DCM03 sensor were connected directly to the LED driver of AFE4490, while the receiver diode was connected directly to a transimpedance amplifier.

The clock signal, control lines and SPI of the AFE4490 system were connected to an external microcontroller. The large integration of the subsystems being used facilitated the development of a measurement system with small external dimensions (ca. $10 \mathrm{~mm} \times 15 \mathrm{~mm}$ ). Thus, the measurement system is suited for wearable electronics.

\section{MEASUREMENT ENVIRONMENT}

\section{Hardware tested}

Experimental studies were carried out using two measurement chains. One of the chains included a transmission sensor, with the signal from the sensor being considered as the reference signal. The second measurement chain included the tested reflectance sensor. Signals from both chains were recorded simultaneously on a PC system with Matlab software. Fig. 6 presents a block diagram of the measurement workstation.

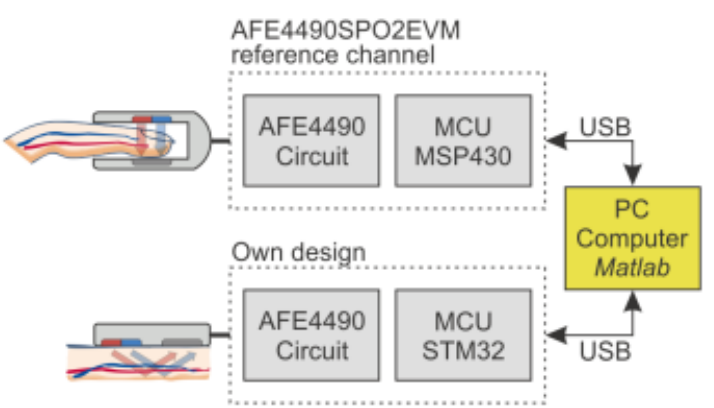

Fig. 6. Block diagram of the measurement testbed.

A Texas Instruments AFE4490SPO2EVM evaluation board was used in the reference measurement chain. The board features an AFE4490 integrated analog front-end, a 16-bit microcontroller of the MSP430 family and a finger worn transmission reflectance sensor. The MSP430 microcontroller was used only as a system for control and communication with a PC system. Communication was established via a USB interface operating as a virtual serial port.

The tested reflectance sensor was connected to a custom evaluation board consisting of an AFE4490 integrated analog front-end and a 32-bit microcontroller of the STM32 family. The STM32 microcontroller was used as a system for control and communication with a PC system as well as an autonomous processing system. Communication with the PC system was established in the same fashion as in the case of AFE4490SPO2EVM board. The STM32 system [31] is a 32-bit microcontroller featuring a Cortex-M4F core and characterized by data storage and calculation powers much higher than those of MSP430. The microcontroller features $1 \mathrm{MB}$ of FLASH storage, $196 \mathrm{kB}$ of RAM 
and a maximum operating frequency of $168 \mathrm{MHz}$. These resources facilitate implementation of appropriate data processing algorithms operating in real time. In addition, the STM32 microcontroller is characterized by a very low energy consumption (ca. $1 \mathrm{~mW} / \mathrm{MHz}$ ) which is very important for a mobile, battery-powered measurement system.

Example placement of the reflectance and the transmission (reference) sensors during the experiments is show in Fig. 7.

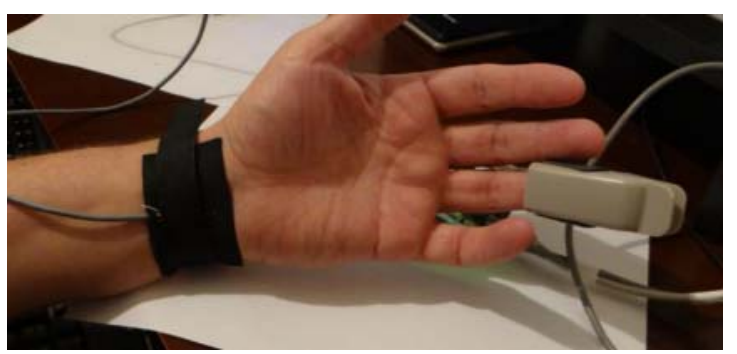

Fig. 7. Placement of the reflectance and the transmission sensors.

The transmission sensor was always placed on the ring finger of the left hand while the reflectance sensor was placed at various sites, including the index finger, top and bottom of the wrist (bottom wrist shown in Fig. 7) and the forehead. Studies with the reflectance sensor placed on the chest and the leg were also attempted; however, the amplitude of the signal at these locations was too low to determine the vital parameters of interest.

The experiments were conducted in line with the methodology approved for research involving people by the Bioethics Commission of the Military Institute of Aviation Medicine in Warsaw. Written informed consent was obtained from participants and the committee approved this procedure.

\section{Data processing tested}

All data collected during the experiment were transferred from the sensor to a computer system. The data were processed on a PC computer with the Matlab software. The data flow scheme diagram is presented in Fig. 8.

Signals from the photodetector were read by a DAQ data acquisition system consisting of the

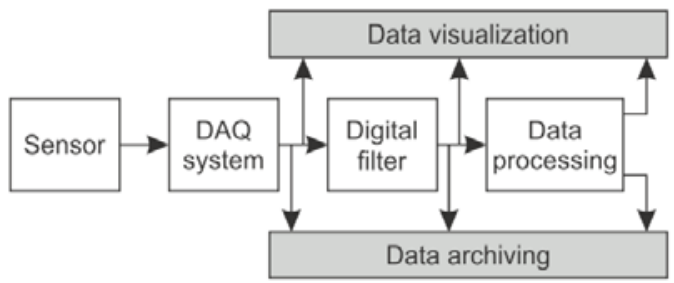

Fig. 8. Diagram of data flow during the experimental studies.
AFE4490 analog front-end and a microcontroller. Sampling frequency was $500 \mathrm{~Hz}$, separately for each diode (RED and IR). Samples of the measured signals were transferred in real time to the computer for digital filtration and preliminary processing. Data from all experiment stages were archived for further processing and off-line analysis.

The received photoplethysmographic signal obtained from the sampling process within the AFE4490 front-end consisted of the pulse wave as the main component together with numerous disturbances. The main disturbances included the slow-variable part due to slight motions of the patient's body (e.g. while breathing), increased pressure of blood under the sensor as well as the high-frequency noise component. In the proposed solution, elimination of the slow-variable component was achieved by means of a procedure described by the following formula:

$$
Y=X^{\prime}-\overline{X_{S A}}+\bar{X}
$$

where: $Y$ - processed signal without the slowvariable component, $\mathrm{X}^{\prime}$ - measured signal including the slow-variable component delayed by half of the moving average period (0.75 s), - moving average with period corresponding to the $40 \mathrm{bpm}$ rate (1.5 s), X - arithmetic mean of signal X. Example results of the method are illustrated in Fig. 9.

In order to eliminate the high-frequency component, an elliptic low-pass filter was used with the order and cut-off frequency determined on an experimental basis. The filter was a fourth-order filter with a bandwidth of $5 \mathrm{~Hz}$. Determination of $\mathrm{SpO} 2$ and HR requires identification of RED and IR signal extremes. One of the identification methods is waveform segmentation, i.e. division into fragments with each fragment encompassing a full waveform period. Minimum and maximum values are determined within each of these segments, corresponding to the extremes of interest. A threshold algorithm with a variable baseline was used in the proposed solution [32]. The baseline consisted of the moving average. Example operation of the segmentation algorithm is shown in Fig. 10. After determination of the extreme values for both PPG signals, the obtained locations are compared in each segment to verify whether the locations of the minima and maxima are identical for both signals. $\mathrm{HR}$ is calculated as the number of periods between the extremes of the same type within one minute.

Extreme signal values are read in sequence to calculate the value of the $\mathrm{R}$ ratio according to formula (3). A more complex method, such as that described in [33], may also be used for calculation of $H R$ values. 


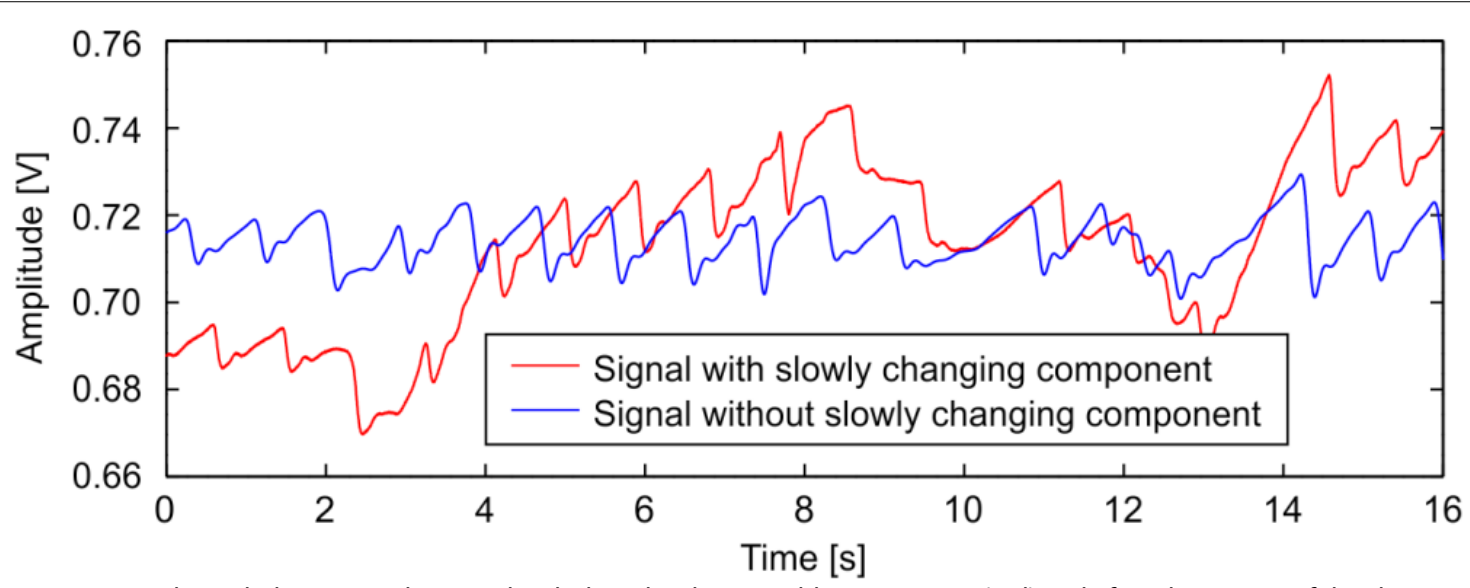

Fig. 9. Photoplethysmographic signal including the slow-variable component (red) and after elimination of the slowvariable component (blue).

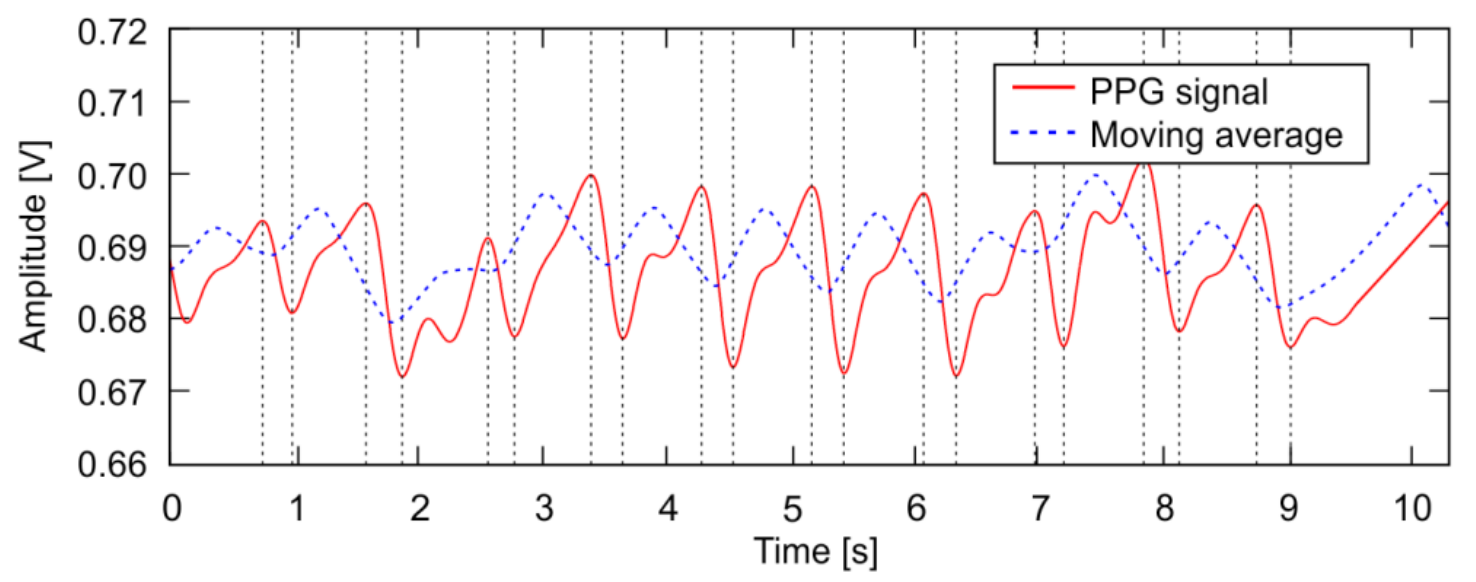

Fig. 10. Segmentation with baseline determined form the moving average.

\section{EXPERIMENTAL RESULTS}

As mentioned previously, the experiments involved different locations of reflectance sensor (DCM03) placement both in stationary conditions and during motion. Signals from all sensors used in the experiments were recorded simultaneously regardless of the conditions. Presented below is a table with summaries of the experiments and the results obtained.

Tab. 1. List of experiments.

\section{Stationary conditions}

No motions were performed in the experiments carried out in stationary conditions. The transmission sensor was used as a reference. Experiments were carried out several times for different sensor placement locations. Only selected results are presented in the graphs.

In the first experiment, the reflectance sensor (DCM03) was placed on a table and pressed with the left index finger. The subject was in a sitting

\begin{tabular}{ccc}
\hline Experiments & Reflectance sensor placement location & Measurement conditions \\
\hline exp1 & Stationary conditions & \\
\hline exp2 & left index finger & subject in standing position \\
\hline exp3 & wrist - below & subject in standing position \\
\hline exp4 & wrist - on top & subject in standing position \\
\hline & forehead & subject in standing position \\
\hline exp5 & The effect of motion on the output signal & \\
\hline exp6 & wrist - below & subject in sitting position, hand moving to and fro \\
\hline exp7 & wrist - below & subject in sitting position, hand moving up and down \\
\hline exp8 & wrist - below & subject in standing position, head moving right and left \\
\hline exp9 & forehead & subject in standing position, performing squats \\
\hline
\end{tabular}


a)

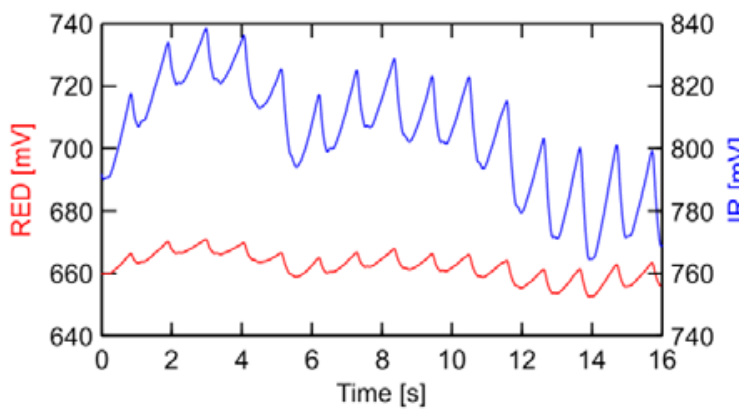

b)

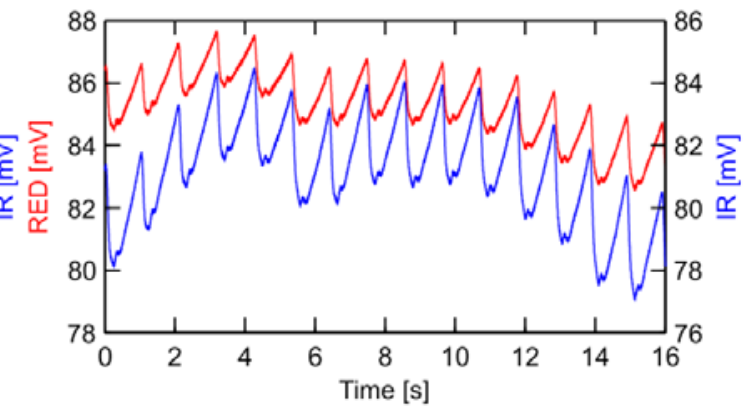

Fig. 11. Signals from RED and IR diodes for the reflectance sensor (a) and the transmission sensor (b), (exp 1).

a)

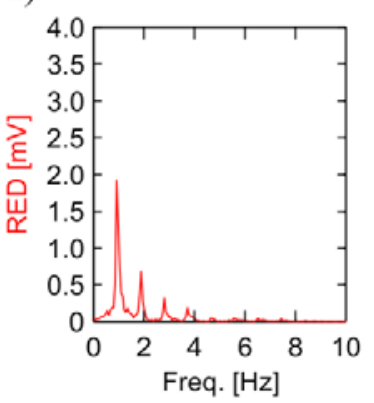

b)

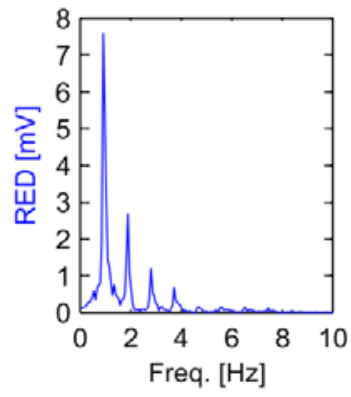

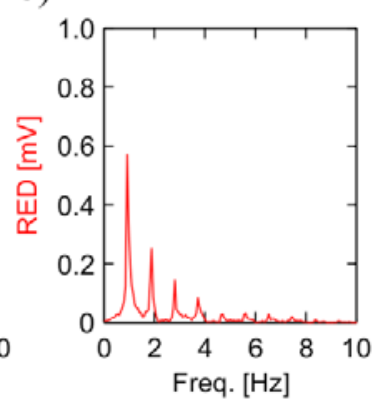

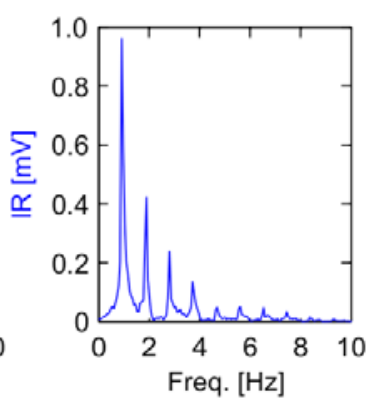

Fig. 12. Amplitude spectrum of signals from RED and IR diodes for the reflectance sensor (a) and the transmission sensor (b), (exp 1).

a)

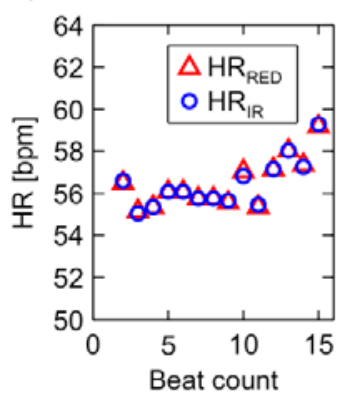

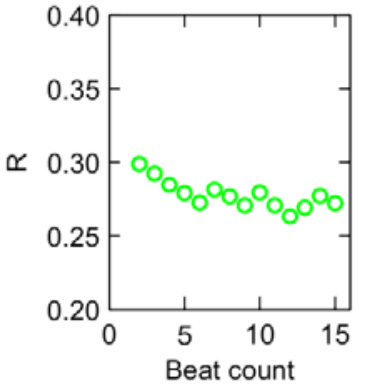

b)

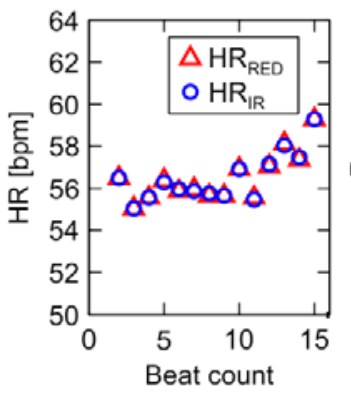

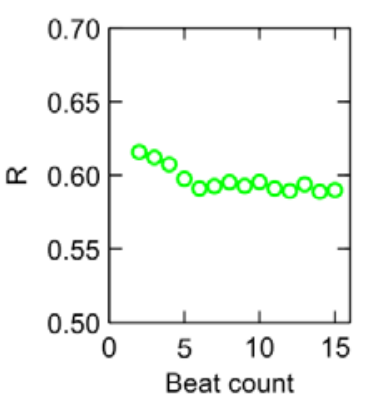

Fig. 13. Heart rate measurement results and $\mathrm{R}$ values for each correctly detected heart beat: (a) reflectance sensor, (b) transmission sensor, (exp 1).

position. The goal of this experiment was to verify the operation of the measurement system. Signals from RED and IR diodes are shown in Fig. 11.

One may notice that the amplitude of the IR diode signal in the reflectance sensor is over $20 \mathrm{mV}$ and is markedly higher than the amplitude of the RED diode signal. In addition, the amplitudes of signals from both diodes for the reflectance sensor are higher than those for the transmission sensor; however, signal amplitude, as such, is not the expected parameter. Moreover, it depends on numerous factors such as sensor pressure strength, sensor placement or amplification in the measurement chain. An appropriately high signal amplitude and, in particular, a high signal-to-noise ratio (SNR) facilitate further data processing and mark- edly affect the accuracy of parameters. One of the expected parameters is heart rate (HR) which may be determined using spectral analysis. Amplitude spectra of signals in exp 1 are shown in Fig. 12.

The component of the highest amplitude, corresponding to the $H R$ value, could be easily identified in all signals. The HR value was also determined by the signal processing method as described in section 3. Another outcome of the data processing was the $\mathrm{R}$ value determined from formula (3). Values obtained from both sensors are shown in Fig. 13. Heart rate values determined from the RED and IR diodes using reflectance and transmission sensors are nearly identical; however, $R$ values differed significantly (typically 0.28 for the reflectance sensor and 0.59 for the transmission sensor). 
The difference is due to different diodes and detectors being used as well as to different locations and methods of sensor placement. This case confirms the well-known fact that calibration is required for a correct determination of $\mathrm{SpO}_{2}$ using each measurement system.

Further studies pertained to the placement of the reflectance sensor in locations that might be potentially useful under dynamic conditions, i.e. locations in which the range of motion would not be limited significantly by sensor placement. In further analysis, signals from RED and IR diodes as well as the respective amplitude spectra are presented for the reflectance sensor only. Results for the sensor placed below the wrist (exp 2) are shown in Fig. 14.

The amplitude of the obtained signal was lower than before, particularly in case of the IR diode.

a)

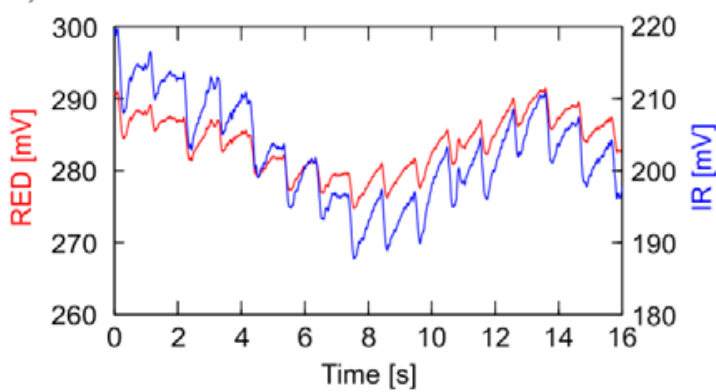

However, the quality of the signal was satisfactory and sufficient for determination of both heart rate and $R$ values. The calculated results of these parameters are shown in Fig. 15.

The reflectance sensor was characterized by a wider range of parameter values as compared to the transmission sensor. In addition, differences in $R$ values were observed in comparison with exp 1, particularly in case of the reflectance sensor. This illustrates how much the $\mathrm{SpO}_{2}$ calculation result is influenced by sensor placement. This is also confirmed by the results obtained in $\exp 3$, where the DCM03 sensor was placed on top of the wrist. The respective graphs are shown in Fig. 16 and Fig. 17.

The signal amplitude recorded from RED and IR diodes of the reflectance sensor is even lower than before (exp 2), with additional significant changes of the constant component making data processb)
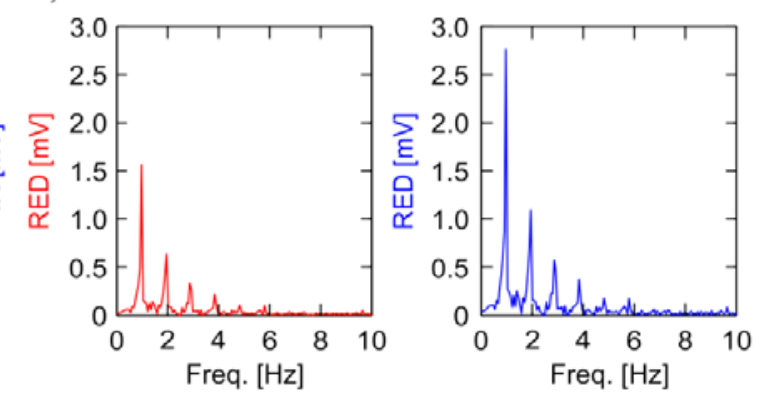

Fig. 14. Results obtained using reflectance sensor: (a) signals from RED and IR diodes, (b) amplitude spectra, (exp 2).

a)

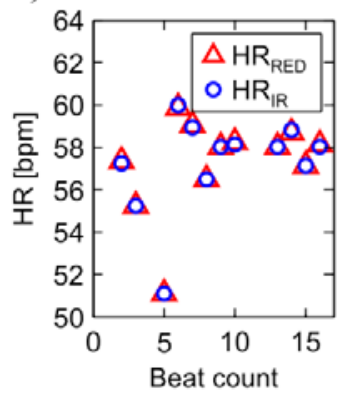

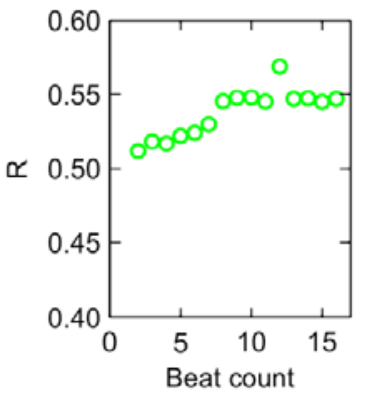

b)

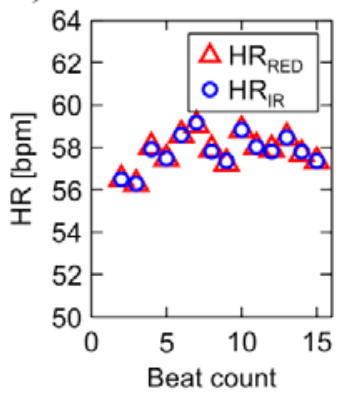

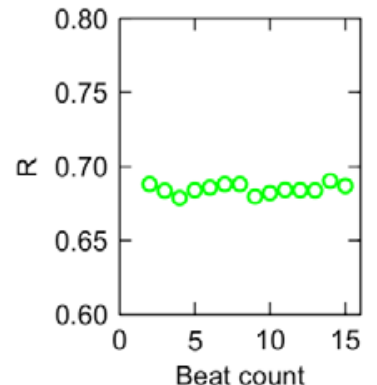

Fig. 15. Heart rate measurement results and $\mathrm{R}$ values for each correctly detected heart beat: (a) reflectance sensor, (b) transmission sensor, $(\exp 2)$.

a)

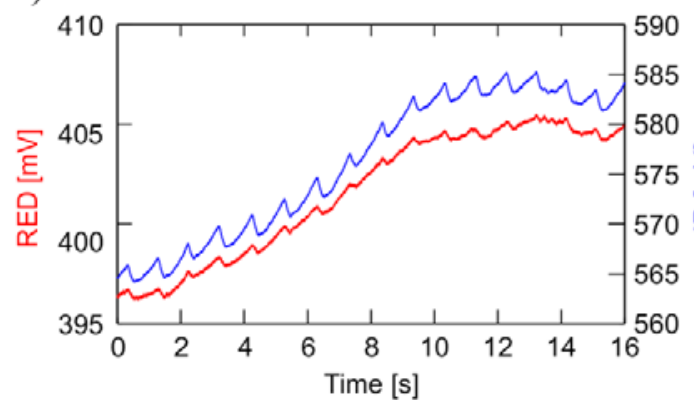

b)

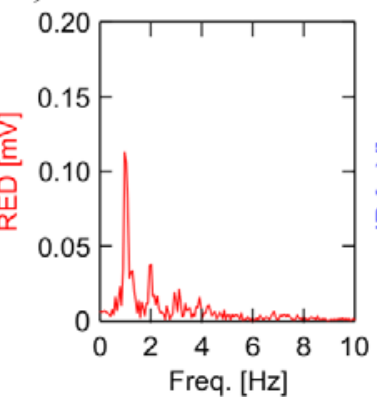

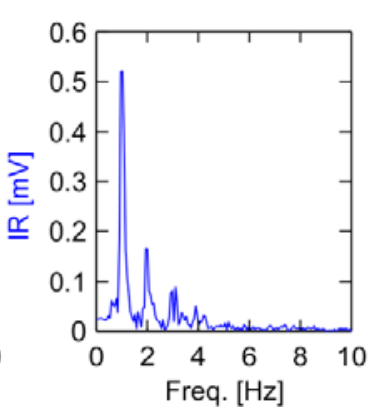

Fig. 16. Results obtained using reflectance sensor: (a) signals from RED and IR diodes, (b) amplitude spectra, (exp 3). 
a)

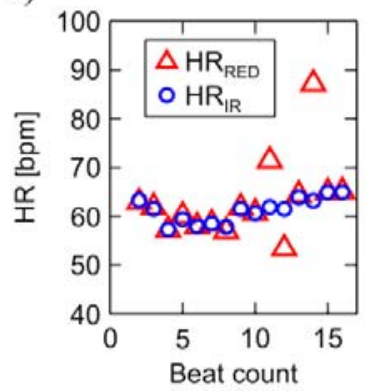

b)

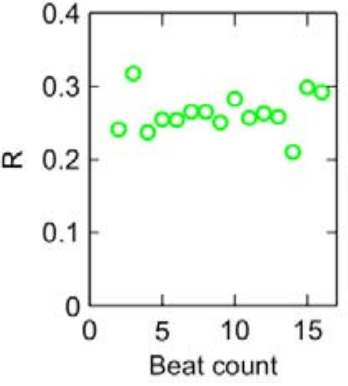

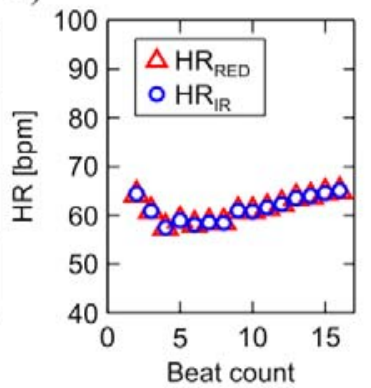

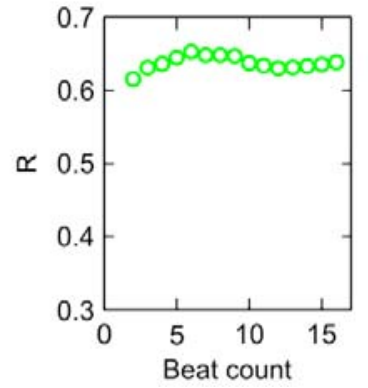

Fig. 17. Heart rate measurement results and R values for each correctly detected heart beat: (a) reflectance sensor, (b) transmission sensor, (exp 3).

a)

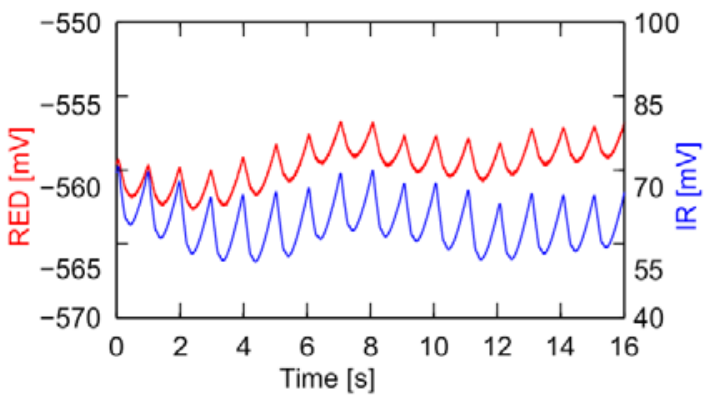

b)

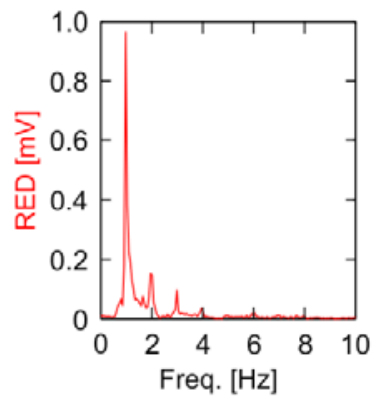

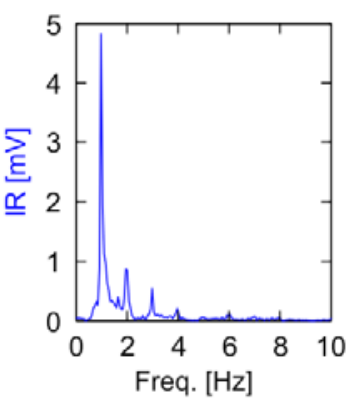

Fig. 18. Results obtained using reflectance sensor: (a) signals from RED and IR diodes, (b) amplitude spectra, (exp 4).

a)

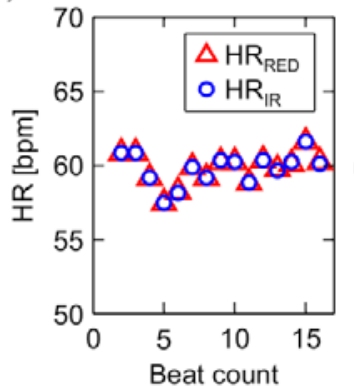

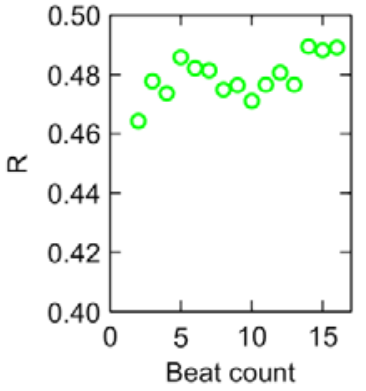

b)

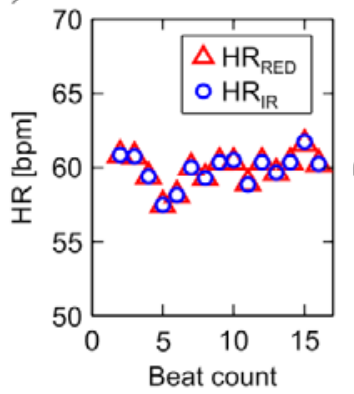

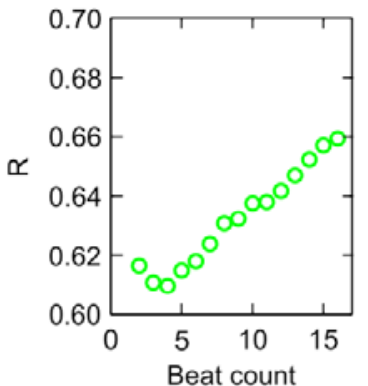

Fig. 19. Heart rate measurement results and $\mathrm{R}$ values for each correctly detected heart beat: (a) reflectance sensor, (b) transmission sensor, (exp 4).

ing more difficult. Also in this case, the signal quality was sufficient for determination of both heart rate and $R$ values.

In case of the reflectance sensor, the forehead was a good measurement location. The results of the experiment (exp 4) are shown in Fig. 18 and Fig. 19.

Signals from RED and IR diodes were not disturbed and characterized by high amplitudes (ca. 2 and $12 \mathrm{mV}$ ), translating into appropriate SNR values. This was also confirmed by the amplitude spectrum (Fig. 18 b).

Heart rates determined using the reflectance and the transmission sensors were characterized by similar values and low deviations from the mean. As in all previous studies, different $R$ values were determined in this experiment.

\section{The effect of motion on the output signal}

In all studies involving the effect of motion, the reflectance sensor was places under the wrist (Fig. 7) or on the forehead. These locations were accepted as most favorable based on previous results. During $\exp 5$, the reflectance sensor was placed under the wrist, while the subject moved their hand to and fro in the horizontal plane. The subject was in a sitting position. The results are shown in Fig. 20 and Fig. 21.

Signals from RED and IR diodes recorded in this experiment were significantly disturbed by the changes in hand position, making the signal changes due to blood flow fluctuations invisible. The proper signal (circled) may be isolated only following appropriate signal processing. The amplitude spectrum (Fig. 20 a) confirmed the presence of the proper 
signal. However, correct determination of HR values was troublesome.

HR values determined from the RED and IR diodes were different for the reflectance sensor while remaining identical for the transmission sensor. In addition, the $\mathrm{R}$ value determined for the reflectance sensor differed significantly at individual time points as evidenced by significant signal disturbances and the inefficiency of the processing method.

Interesting results were obtained in exp 6 in which the subject performed alternating upward and downward motions with their arm in a standing position. The largest changes in the signals from RED and IR diodes (Fig. 22) were due to the changes in blood pressure at the measurement site (wrist). The useful signal had an amplitude nearly 60 times lower than the changes in the constant component due to the arm motion. In this case, the pulse signal was difficult to notice even in the amplitude spectrum.

For this type of motion, the efficacy of processing algorithms is insufficient for the reflectance sensor. The results are shown in Fig. 23.

HR values determined from RED and IR diodes for the reflectance sensor were significantly different, with additional significant deviations from the mean value. In case of the transmission sensor, the $H R$ values were determined correctly. $R$ values were determined correctly only in the case of the transmission sensor.

In the next experiment (exp 7), the subject was performing squats from a standing position. The results are shown in Fig. 24 and Fig. 25.

The amplitude of the RED and IR diode signals changed mainly along the squatting rhythm, a)

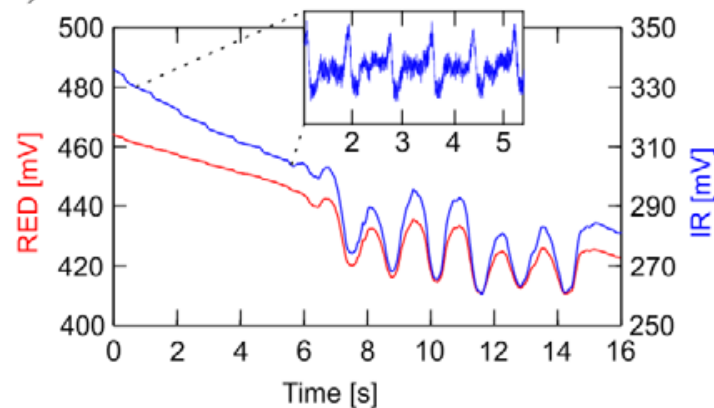

b)

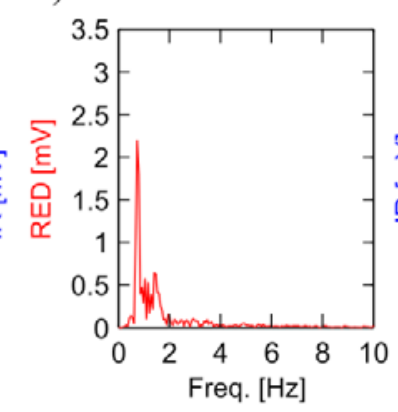

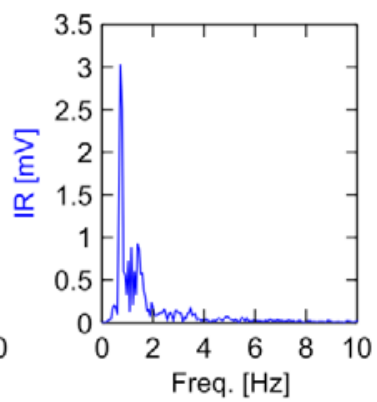

Fig. 20. Results obtained using reflectance sensor: (a) signals from RED and IR diodes, (b) amplitude spectra, (exp 5).

a)

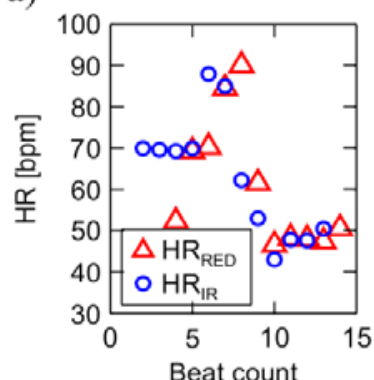

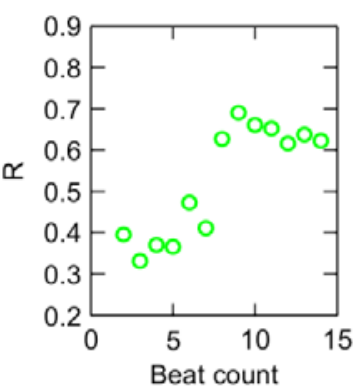

b)

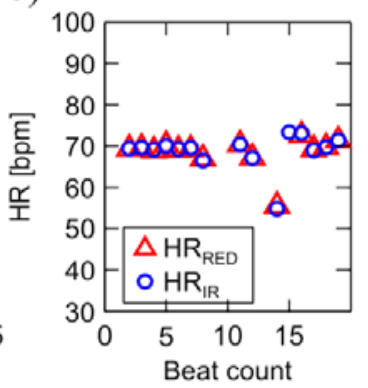

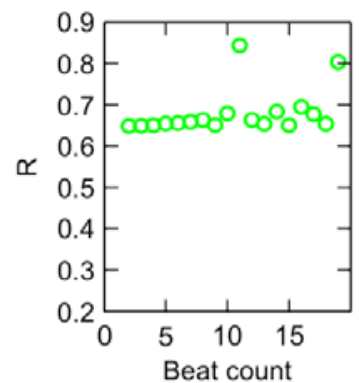

Fig. 21. Heart rate measurement results and $\mathrm{R}$ values for each correctly detected heart beat: (a) reflectance sensor, (b) transmission sensor, (exp 5).

a)

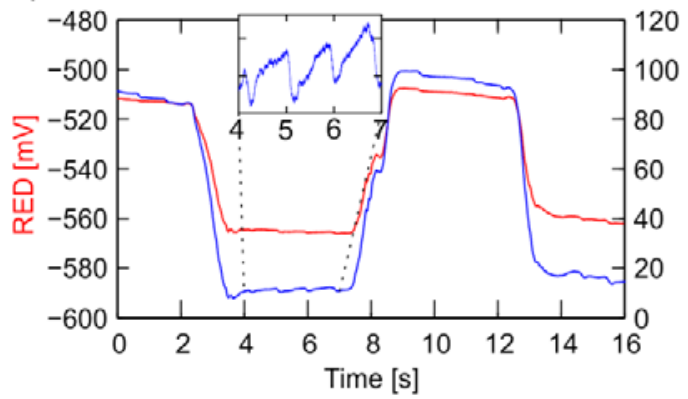

b)

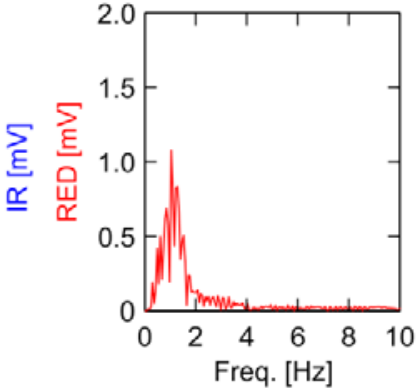

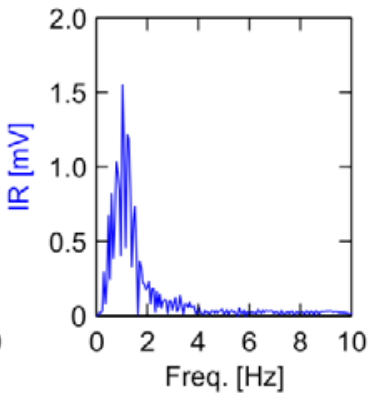

Fig. 22. Results obtained using reflectance sensor: (a) signals from RED and IR diodes, (b) amplitude spectra, (exp 6). 
a)

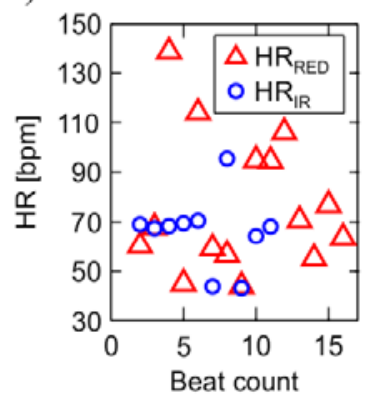

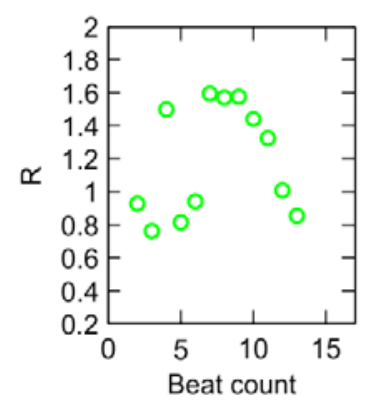

b)

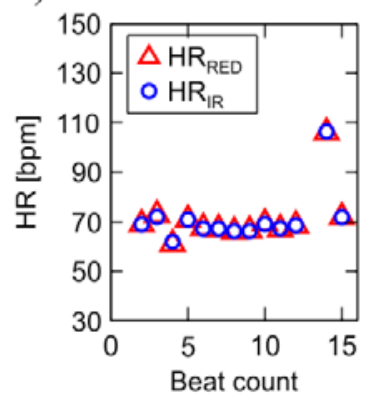

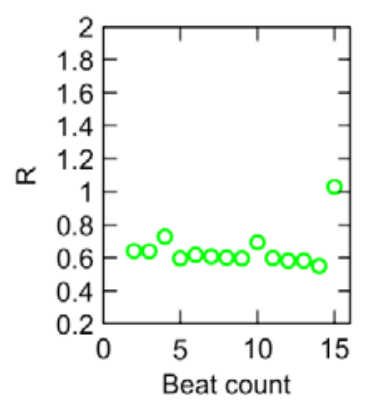

Fig. 23. Heart rate measurement results and R values for each correctly detected heart beat: (a) reflectance sensor, (b) transmission sensor, (exp 6).

a)

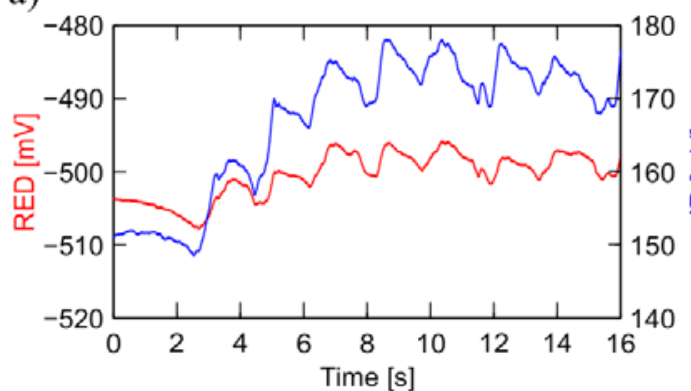

b)

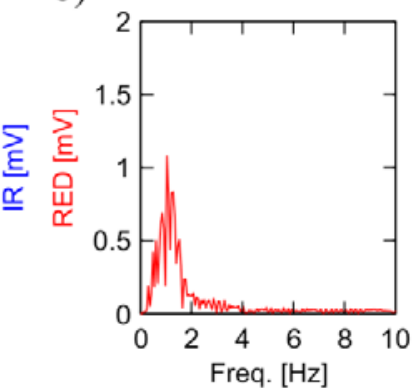

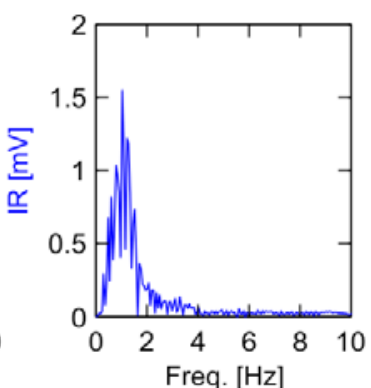

Fig. 24. Results obtained using reflectance sensor: (a) signals from RED and IR diodes, (b) amplitude spectra, (exp 7).

a)

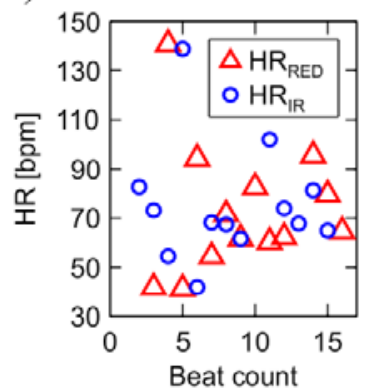

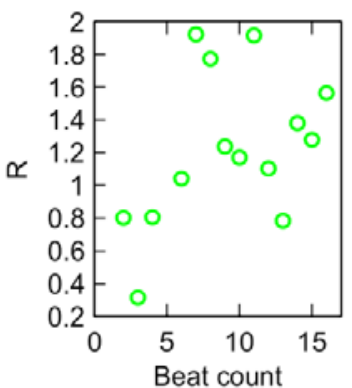

b)

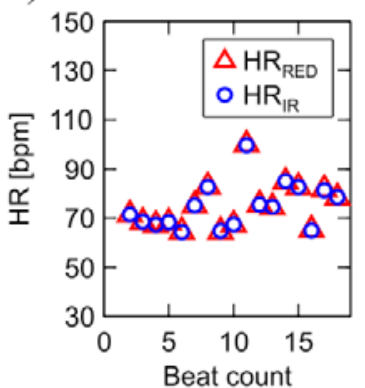

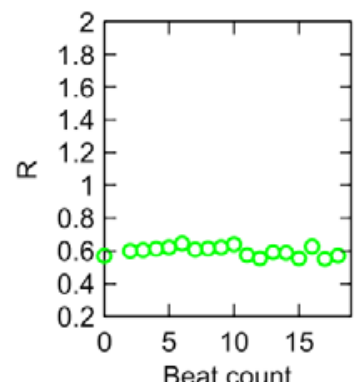

Fig. 25. Heart rate measurement results and $\mathrm{R}$ values for each correctly detected heart beat: (a) reflectance sensor, (b) transmission sensor, (exp 7).

making it very difficult to detect the useful signal. However, the HR component could be noticed in the amplitude spectrum (Fig. 24b). The efficacy of data processing algorithms was insufficient in case of the reflectance sensor. The obtained HR and R (Fig. 25a) values were very discrepant. At the same time, satisfactory results were obtained using the transmission sensor.

In case of the experiment involving the subject's motion, the reflectance sensor was also placed on the forehead, while the subject moved their head slowly to the left and to the right $(\exp 8)$. The results are shown in Fig. 26 and Fig. 27.

The slow-changing component of the signal from the RED and IR diodes changed as the result of the motion (Fig. 26a); however, the change was slight enough for a successful determination of $H R$ and $R$ rates. Fig. 27a shows high conformity of HR values determined from the RED and IR signals as well as an undisturbed $R$ value for the reflectance sensor measurements. The correctness of data processing algorithms is confirmed by the results obtained using the transmission sensor shown in Fig. 27b.

The last experiment (exp 9) involved the subject performing squats with a reflectance sensor placed on the forehead as well. The results are shown in Fig. 28 and Fig. 29.

In this case, the variability of the amplitude of signals from the RED and IR diodes (Fig. 28 a) due to the subject's motion was much larger than before (exp 8); however, the useful signal remained identifiable. This is well seen in the amplitude spectrum (Fig. 28b). However, HR and R values determined for the reflectance sensor (Fig. 29a) are characterized by a greater variability than those determined using the transmission sensor (Fig. 29b). 
a)

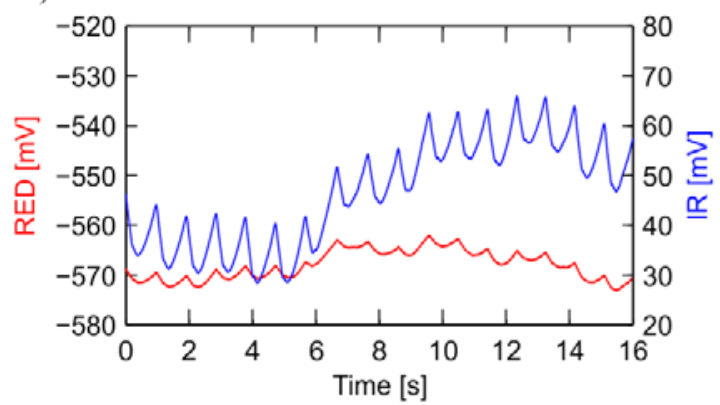

b)

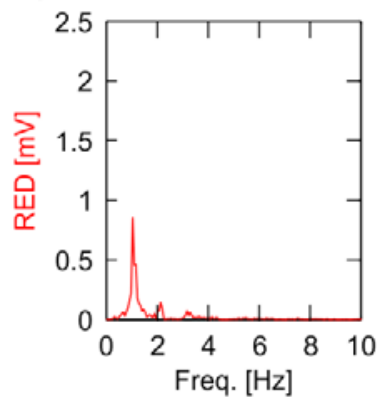

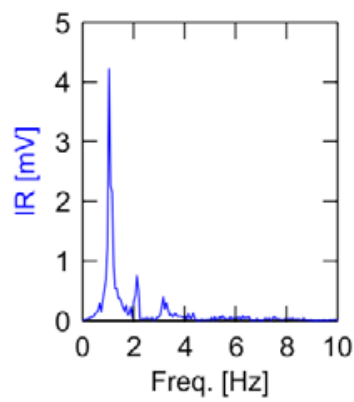

Fig. 26. Results obtained using reflectance sensor: (a) signals from RED and IR diodes, (b) amplitude spectra, (exp 8).

a)

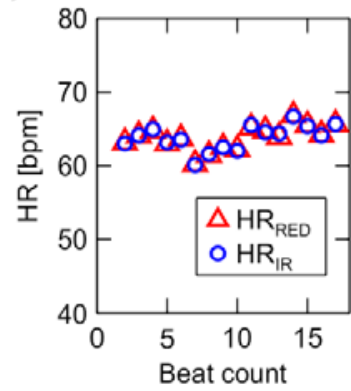

b)

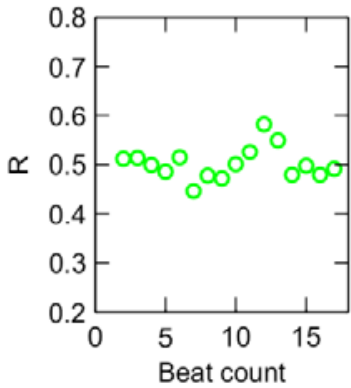

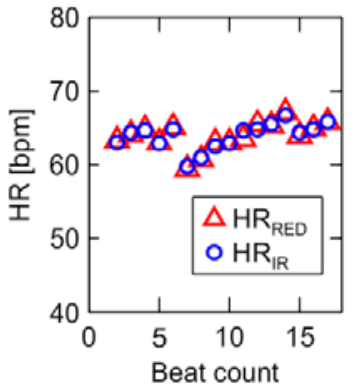

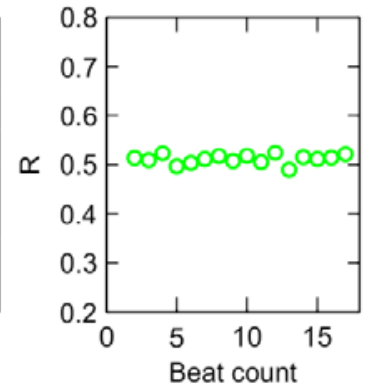

Fig. 27. Heart rate measurement results and $\mathrm{R}$ values for each correctly detected heart beat: (a) reflectance sensor, (b) transmission sensor, (exp 8).

a)

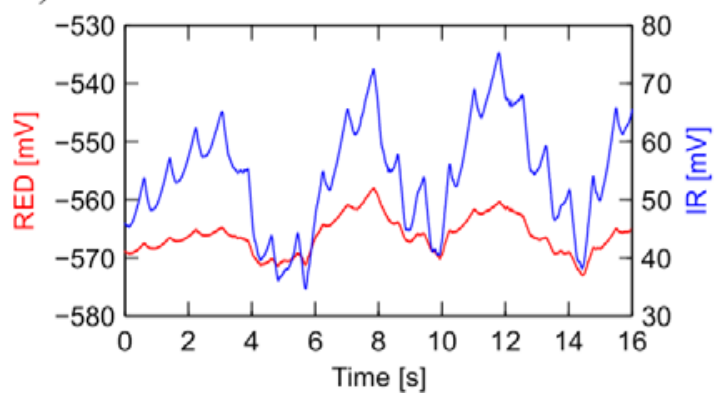

b)

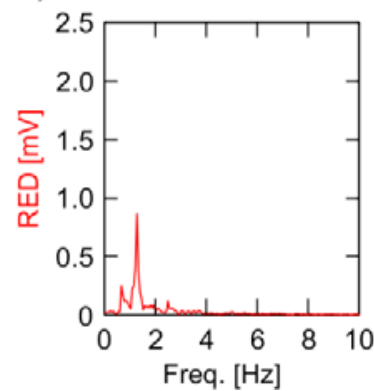

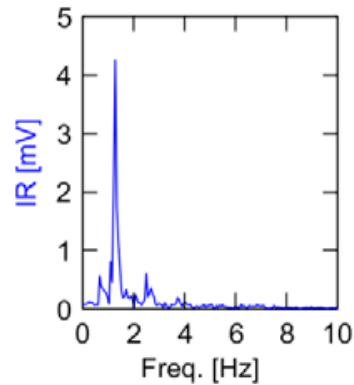

Fig. 28. Results obtained using reflectance sensor: (a) signals from RED and IR diodes, (b) amplitude spectra, (exp 9).

a)

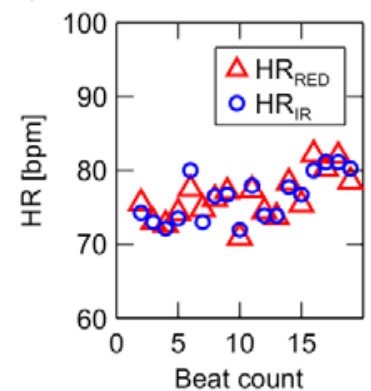

b)

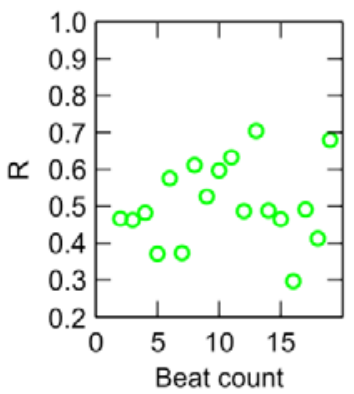

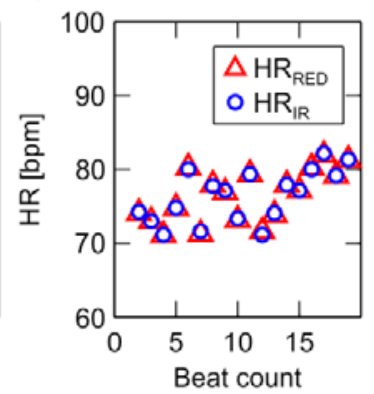

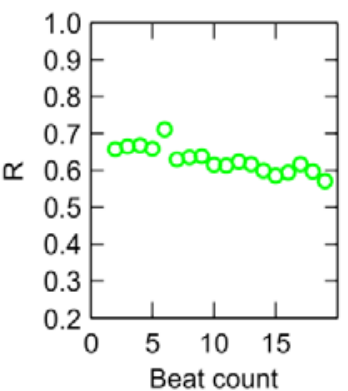

Fig. 29. Heart rate measurement results and R values for each correctly detected heart beat: (a) reflectance sensor, (b) transmission sensor, (exp 9). 


\section{DISCUSSION AND CONCLUSION}

Experiments involving several selected motion activities were performed with the objective to verify the efficacy of plethysmographic signal measurements under dynamic conditions. In each experiment, the amplitudes of the useful signal (A sig.) and signal disturbance (A dis.) were estimated and $H R$ and $R$ values were determined. $H R$ was determined by two methods: in the frequency domain (HR FFT) as well as in the temporal domain (HR alg). All calculations were carried out separately for the red (LR) and infrared (LI) diodes. The obtained results are listed in Table 2.

The analysis of the graphs presented above, together with the experimental results and Table 2 , show that the amplitude of the useful signal as read from the infrared diode is higher than that read from the red diode. In addition, the signal amplitude is usually larger for the transmission sensor as compared to the reflectance sensor. This is due, among other factors, to the fact that light in the tissues is scattered more in the longitudinal direction (i.e. backwards) than in the transverse direction as well as to the lower changes in the constant component observed in case of the transmission sensor, allowing for a higher amplification setting in the system. A high amplification is not possible in case of the reflectance sensors due to very large variations in the constant component that might possibly lead to the transgressions of the $A / C$ processing range. In the experiments involving specific motions, signal changes due to the respective motions - understood as signal disturbances ( $A$ dis.), were much higher (up to several dozen times) than the useful signal (A sig.). This made the determination of $H R$ and $R$ values much more difficult.

As shown in the table above, HR values determined under stationary measurement conditions (subjects not moving) were similar regardless of the calculation method (HR FFT vs. HR alg.), sensor type (reflectance vs. transmission) and LED diode (LR vs. LI). This confirms the efficacy of the tested measurement and data processing method. $R$ values remained similar only in case of the transmission sensor. For the reflectance sensor under stationary conditions, $\mathrm{R}$ values ranged from 0.26 to 0.54 . This shows that the measurement of $\mathrm{SpO}_{2}$ using the reflectance method is very difficult and largely depends on the site and method of sensor placement. Therefore, the measurement system should be calibrated after each sensor placement. For the measurement conditions involving subject's motion, signal disturbances were even larger and a proper determination of heart rate from the reflectance sensor signal was very difficult. Significant differences are observed between HR FFT and HR alg. values. In case of motion studies, the $R$ values determined from the reflectance method should be considered as incorrect (with the exception of exp. 8).

As demonstrated by the experimental studies, the reflectance method may be used in the measurement of the plethysmographic signal also under dynamic conditions. However, this requires appropriate measurement and data processing methods. Heart rate is an additional parameter that may be determined correctly with the use of plethysmographic sensors.

Tab. 2. Experimental results.

\begin{tabular}{|c|c|c|c|c|c|c|c|c|c|c|c|c|c|c|c|c|c|c|c|c|}
\hline \multirow{4}{*}{$\begin{array}{c}\text { Experi- } \\
\text { ment }\end{array}$} & \multicolumn{10}{|c|}{ Reflectance sensor DCMO3 } & \multicolumn{10}{|c|}{ Transmission sensor (as reference) } \\
\hline & \multicolumn{2}{|c|}{ A sig. [mV] } & \multicolumn{2}{|c|}{ A dis. [mV] } & \multirow{2}{*}{\multicolumn{2}{|c|}{$\begin{array}{c}\mathrm{HR} \\
\mathrm{FFT}[\mathrm{bpm}]\end{array}$}} & \multirow{2}{*}{\multicolumn{2}{|c|}{$\begin{array}{c}\mathrm{HR} \\
\text { alg. [bpm] }\end{array}$}} & \multirow{3}{*}{$\begin{array}{c}\mathrm{R} \\
\text { mean } \\
{[\%]}\end{array}$} & \multirow{3}{*}{$\begin{array}{l}\mathrm{R} \\
\text { std } \\
{[\%]}\end{array}$} & \multicolumn{2}{|c|}{ A sig. [mV] } & \multicolumn{2}{|c|}{ A dis. [mV] } & \multirow{2}{*}{\multicolumn{2}{|c|}{$\begin{array}{c}\text { HR } \\
\text { FFT [bpm] }\end{array}$}} & \multirow{2}{*}{\multicolumn{2}{|c|}{$\begin{array}{c}\text { HR } \\
\text { alg. [bpm] }\end{array}$}} & \multirow{3}{*}{$\begin{array}{c}\mathrm{R} \\
\text { mean } \\
{[\%]}\end{array}$} & \multirow{3}{*}{$\begin{array}{c}\mathrm{R} \\
\text { std } \\
{[\%]}\end{array}$} \\
\hline & \multirow{2}{*}{ LR } & \multirow[t]{2}{*}{ LI } & \multirow[t]{2}{*}{$\mathrm{LR}$} & \multirow[t]{2}{*}{ LI } & & & & & & & \multirow[t]{2}{*}{$\mathrm{LR}$} & \multirow[t]{2}{*}{$\mathrm{LI}$} & \multirow[t]{2}{*}{$\mathrm{LR}$} & \multirow[t]{2}{*}{$\mathrm{LI}$} & & & & & & \\
\hline & & & & & $\mathrm{LR}$ & LI & $\mathrm{LR}$ & LI & & & & & & & LR & LI & LR & LI & & \\
\hline Ex1 & 5 & 20 & - & - & 54.9 & 54.9 & 56.5 & 56.5 & 0.28 & 0.009 & 2 & 3 & - & - & 54.9 & 54.9 & 56.5 & 56.5 & 0.60 & 0.009 \\
\hline Ex2 & 5 & 8 & - & - & 58.6 & 58.6 & 66.6 & 66.6 & 0.54 & 0.016 & 4 & 6 & - & - & 58.6 & 58.6 & 57.8 & 57.8 & 0.68 & 0.003 \\
\hline Ex3 & 1 & 3 & - & - & 58.6 & 62.3 & 62.9 & 61.2 & 0.26 & 0.026 & 3 & 4 & - & - & 62.3 & 62.3 & 61.3 & 61.3 & 0.64 & 0.010 \\
\hline Ex4 & 2 & 12 & - & - & 58.6 & 58.6 & 59.8 & 59.8 & 0.48 & 0.007 & 40 & 50 & - & - & 58.6 & 58.6 & 59.9 & 59.9 & 0.64 & 0.018 \\
\hline Ex5 & 0.5 & 2 & 20 & 30 & 43.9 & 43.9 & 67.4 & 63.0 & 0.53 & 0.136 & 20 & 25 & - & - & 69.6 & 69.6 & 81.8 & 80.0 & 0.68 & 0.053 \\
\hline Ex6 & 0.8 & 1.5 & 60 & 80 & 62.3 & 62.3 & 76.4 & 65.9 & 1.19 & 0.335 & 15 & 25 & 50 & 100 & 69.6 & 69.6 & 81.1 & 81.1 & 0.63 & 0.113 \\
\hline Ex7 & 0.7 & 1 & 5 & 8 & 65.9 & 65.9 & 85.5 & 84.1 & 1.32 & 0.589 & 15 & 25 & 40 & 80 & 69.6 & 69.6 & 77.1 & 77.0 & 0.60 & 0.029 \\
\hline Ex8 & 3 & 10 & 10 & 30 & 62.3 & 62.3 & 63.8 & 63.8 & 0.50 & 0.032 & 30 & 50 & - & - & 62.3 & 62.3 & 63.9 & 63.9 & 0.54 & 0.014 \\
\hline Ex9 & 1 & 6 & 12 & 30 & 76.9 & 76.9 & 76.8 & 77.0 & 0.51 & 0.105 & 25 & 40 & 50 & 60 & 76.9 & 76.9 & 76.8 & 76.8 & 0.62 & 0.035 \\
\hline \multicolumn{21}{|c|}{ LR - red diode, LI - infrared diode } \\
\hline \multicolumn{21}{|c|}{ A sig. - amplitude of the useful signal, A dis. - amplitude of disturbances } \\
\hline \multicolumn{21}{|c|}{ HR FFT - heart rate from fast Fourier transform frequency analysis, HR alg. - heart rate from time analysis algorithm } \\
\hline \multicolumn{21}{|c|}{$R$ mean - mean value of $R, R$ std - standard deviation of $R$} \\
\hline
\end{tabular}




\section{AUTHORS' DECLARATION:}

Study Design: Tadeusz Sondej, Krzysztof Różanowski, Dominik Sondej; Data Collection: Tadeusz Sondej, Krzysztof Różanowski, Dominik Sondej; Manuscript Preparation: Tadeusz Sondej, Krzysztof Różanowski, Dominik Sondej; Funds Collection: Tadeusz Sondej, Krzysztof Różanowski, Dominik Sondej. The Authors declare that there is no conflict of interest.

\section{REFERENCES}

1. Mendelson $\mathrm{Y}$, Ochs BD. Noninvasive pulse oximetry utilizing skin reflectance photoplethysmography. IEEE Transactions on Biomedical Engineering 1988; 35(10): 798-805.

2. Mendelson $\mathrm{Y}$, Dao DK, Chon KH. Multi-channel pulse oximetry for wearable physiological monitoring. Body Sensor Networks (BSN), 2013 IEEE International Conference on 2013: 1-6.

3. Kejia L, Warren SA. Wireless reflectance pulse oximeter with digital baseline control for unfiltered photoplethysmograms. IEEE Transactions on Biomedical Circuits and Systems 2012; 6(3): 269-278.

4. Seung-Min Park, Jun-Yeup Kim, Kwang-Eun Ko, In-Hun Jang, Kwee-Bo Sim. Real-Time Heart Rate Monitoring System based on Ring-Type Pulse Oximeter Sensor. Journal of Electrical Engineering and Technology 2013; 8(2): 376-384.

5. Wong MYM, Pickwell-MacPherson E, Zhang YT. Contactless and continuous monitoring of heart rate based on photoplethysmography on a mattress. Physiol. Meas. 2010; 31: 65-1074.

6. Budidha K, Kyriacou PA. The human ear canal: investigation of its suitability for monitoring photoplethysmographs and arterial oxygen saturation. Physiol. Meas. 2014; 35: 111-128.

7. Cheng-Yang Huang, Ming-Che Chan, Chien-Yue Chen, Bor-Shyh Lin. Novel Wearable and Wireless Ring-Type Pulse Oximeter with Multi-Detectors. Sensors 2014; 14(9): 17586-17599.

8. Haahr RG, Duun SB, Toft MH, Belhage B, Larsen J, Birkelund K, Thomsen EV. An Electronic Patch for Wearable Health Monitoring by Reflectance Pulse Oximetry. IEEE Transactions on Biomedical Circuits and Systems 2012; 6(1): 45-53.

9. Wei Chen, Ayoola I, Oetomo SB, Feijs L. Non-invasive blood oxygen saturation monitoring for neonates using reflectance pulse oximeter. Design, Automation \& Test in Europe Conference \& Exhibition (DATE) 2010: 1530-1535.

10. Petersen Christian L, Chen TP, Mark Ansermino J, Dumont Guy A. Design and Evaluation of a Low-Cost Smartphone Pulse Oximeter. Sensors 2013; 13(12): 16882-16893.

11. Stojanovic R, Karadaglic D. Design of an Oximeter Based on LED-LED Configuration and FPGA Technology. Sensors 2013; 13(1): 574-586.

12. Toshiyo Tamura, Yuka Maeda, Masaki Sekine, Masaki Yoshida. Review: Wearable Photoplethysmographic Sensors-Past and Present. Electronics 2014; 3: 282-302.

13. Clarke GWJ, Chan ADC, Adler A. Effects of motion artifact on the blood oxygen saturation estimate in pulse oximetry. Medical Measurements and Applications (MeMeA), 2014 IEEE International Symposium on 2014: 1-4.

14. Ashoka Reddy K, Boby G, Jagadeesh K, V. Use of Fourier Series Analysis for Motion Artifact Reduction and Data Compression of Photoplethysmographic Signals. IEEE Trans on Instrumentation and Measurement 2009; 58(5): 1706-1711.

15. Yash Pole, Evolution of the pulse oximeter. International Congress Series 2002; 1242: 137-144.

16. Han-Wook Lee, Ju-Won Lee, Won-Geun Jung, Gun-Ki Lee, The Periodic Moving Average Filter for Removing Motion Artifacts from PPG Signals. International Journal of Control, Automation, and Systems 2007; 5(6): 701-706.

17. Lee CM, Zhang YT. Reduction of Motion Artifacts from Photoplethysmographic Recordings Using a Wavelet Denoising Approach. IEEE Transactions on Biomedical Engineering 2003; 50(3): 194-195.

18. Jensen T, Duun S, Larsen J, Haahr RG, Toft MH, Belhage B, Thomsen EV. Independent component analysis applied to pulse oximetry in the estimation of the arterial oxygen saturation (SpO2) - a comparative study. Engineering in Medicine and Biology Society, Annual International Conference of the IEEE 2009: 4039-4044.

19. Gibbs P, Asada HH. Reducing motion artifact in wearable bio-sensors using MEMS accelerometers for active noise cancellation. Proceedings of the 2005 American Control Conference 2005; 3: 1581-1586.

20. Yousefi R, Nourani M, Ostadabbas S, Panahi I. A Motion-Tolerant Adaptive Algorithm for Wearable Photoplethysmographic Biosensors. IEEE Journal of Biomedical and Health Informatics 2014; 18(2): 670-681.

21. Goldman JM, Petterson MT, Kopotic RJ, Barker SJ. Masimo Signal Extraction Pulse Oximetry. Journal of Clinical Monitoring and Computing 2000; 16(7): 475-483. 
22. Yong-Sheng Yan, Yuan-Ting Zhang, An Efficient Motion-Resistant Method for Wearable Pulse Oximeter. IEEE Transactions on Information Technology in Biomedicine 2008; 12(3): 399-405.

23. Ming-Zher Poh, Swenson NC, Picard RW. Motion-tolerant magnetic earring sensor and wireless earpiece for wearable photoplethysmography. IEEE Transactions on Information Technology in Biomedicine 2010; 14(3): 786-794.

24. Patterson JAC, Guang-Zhong Yang, Ratiometric Artifact Reduction in Low Power Reflective Photoplethysmography. IEEE Transactions on Biomedical Circuits and Systems 2011; 5(4): 330-338.

25. Mendelson Y. Pulse oximetry: theory and applications for noninvasive monitoring. Clinical Chemistry 1992; 38(9): 1601-1607.

26. Shafique M, Kyriacou PA. Photoplethysmographic signals and blood oxygen saturation values during artificial hypothermia in healthy volunteers. Physiol. Meas. 2012; 33: 2065-2078.

27. Duun SB, Haahr RG, Birkelund K, Thomsen EV. A Ring-Shaped Photodiode Designed for Use in a Reflectance Pulse Oximetry Sensor in Wireless Health Monitoring Applications. IEEE Sensors Journal 2010; 10(2): 261-268.

28. Zaman T, Kyriacou PA, Pal SK. Free flap pulse oximetry utilizing reflectance photoplethysmography. Engineering in Medicine and Biology Society (EMBC), 35th Annual International Conference of the IEEE, 2013: 4046-4049.

29. APMKorea, DCM03 Data Sheet, http://www.apmkr.com/spo2_oximeter_blood_sensor.htm, (accessed on 25 May 2014).

30. Texas Instruments, Integrated Analog Front-End for Pulse Oximeters, AFE4490 Data Sheet, SBAS602D - December 2012-Revised May 2013.

31. STMicroelectronics, STM32F405xx Data Sheet, DocID 022152, Rev 4, June 2013.

32. Bołtrukiewicz M, Cysewska-Sobusiak A. Algorytmy kondycjonowania sygnału fotopletyzmograficznego (The algorithms of photoplethysmographic signal conditioning). Pomiary, Automatyka, Kontrola 2005; 9: 22-25.

33. Piotrowski Z, Rozanowski K. Robust Algorithm for Heart Rate (HR) Detection and Heart Rate Variability (HRV) Estimation. Acta Physica Polonica A. 2010; 118(1): 131-135.

\section{ACKNOWLEDGEMENTS}

The views, opinions, and findings contained in this article are our own and should not be construed as an official Polish Air Force position, policy, or decision, unless so designated by other official documentation.

Cite this article as: Sondej T, Różanowski K, Sondej D. Reflectance Method for a Wearable Photoplethysmographic sensor - Experimental Evaluation. Pol J Aviat Med Bioeng Psychol 2016; 22(1): 5-20. DOI: 10. 13174/ pj ambp. 30. 12. 2016. 01 